\title{
Unconventional players on the striated muscle field: microRNAs, signaling pathways and epigenetic regulators
}

\author{
Giorgia Giacomazzi ${ }^{1,3}$, Maurilio Sampaolesi ${ }^{1,2,3}$, Mattia Quattrocelli ${ }^{1,3, *}$ \\ ${ }^{1}$ Translational Cardiomyology Lab, Embryo and Stem Cell Biology, Dept Development \\ and Regeneration, KU Leuven, Belgium. \\ ${ }^{2}$ Division of Human Anatomy, Dept of Public Health, Experimental and Forensic \\ Medicine, University of Pavia, Italy. \\ ${ }^{3}$ Interuniversity Institute of Myology (IIM), Italy. \\ *Correspondence to: \\ Mattia Quattrocelli, PhD \\ Translational Cardiomyology Lab \\ Dept Development and Regeneration \\ KU Leuven \\ Herestraat 49 - O\&N4 - bus 814 \\ 3000 Leuven \\ Belgium \\ mattia.quattrocelli@med.kuleuven.be
}

Keywords: striated muscle, regeneration, non-genetic factors, microRNAs, signaling pathways, epigenetic factors.

Word count: 3238 (excluding Abstract and References) 


\begin{abstract}
Striated muscle regeneration holds an intrinsic complexity governed by many orchestrated events. When the fine balance of regulatory machineries is under strain, the homeostatic conditions are lost and degeneration starts to occur. This is the case for inherited and acquired diseases of both cardiac and skeletal muscles. A wide range of factors is currently under scrutiny for better understanding the details underlying de-/regeneration processes, of both genetic and non-genetic nature. This review focuses on three classes of non-genetic factors regulating striated muscle regeneration, i.e. microRNAs, signaling pathways and epigenetic regulators.
\end{abstract}




\section{List of abbreviations}

\begin{tabular}{|c|c|}
\hline DNMTs & DNA methyltransferases \\
\hline Ezhl/2 & Enhancer Of Zeste Homolog 1/2 \\
\hline $\mathrm{HGF}$ & Hepatocyte growth factor \\
\hline $\mathrm{H} 3 \mathrm{~K} 4 \mathrm{me} 3$ & Trimethylation of lysine 4 on histone 3 \\
\hline H3K27me3 & Trimethylation of lysine 27 on histone 3 \\
\hline H3K9me2 & Dimethylation of lysine 9 on histone 3 \\
\hline IGF & Insulin growth factor \\
\hline IGFR & Insulin growth factor receptor \\
\hline IL-6 & Interleukin 6 \\
\hline MAGIC-F1 & Met-activating genetically improved chimeric factor 1 \\
\hline MDs & Muscular Dystrophies \\
\hline MEF2 & Myocyte enhancer factor-2 \\
\hline miRNAs & MicroRNAs \\
\hline MLP & Muscle LIM protein \\
\hline MRFs & Myogenic regulatory factors \\
\hline MyoD & Myogenic differentiation 1 \\
\hline RISC & Rna-induced silencing complex \\
\hline SRF & Serum response factor \\
\hline TCF-complex & Trancription factor complex \\
\hline UTR & Untranslated region \\
\hline
\end{tabular}




\section{Introduction}

During the past years, accumulating evidence in a number of tissues has shed more light on the role of non-genetic factors in homeostasis and regeneration of striated muscles, i.e. cardiac and skeletal muscles. These unconventional factors are becoming increasingly intriguing because of their fine timely tuning on downstream conventional genetic factors. Non-genetic factors include short non-coding RNAs, such as microRNAs (miRNAs), ligand-triggered signaling cascades and epigenetic factors, such as histone remodelers and DNA methyltransferases. This review will therefore concisely report on the role of non-genetic factors from those three classes in striated muscle de-/regeneration.

\section{MiRNAs and muscle regeneration}

MiRNAs are short non-coding RNAs that regulate gene expression at post-transcriptional level. MiRNAs are encoded in the genome either as individual transcriptional units, or often as intergenic clusters of several miRNAs. However, growing evidence has shown that a number of miRNAs are enclosed in the intronic sequences of other genes and are therefore transcribed along with the coding genes [1,2]. In the nucleus, miRNAs are generally transcribed by RNA polymerase II in primary transcripts called pri-miRs, subsequently cleaved by the microprocessor complex into shorter precursor molecules called pre-miRs [3]. Pre-miRs are then transported into the cytosol, where they are further cleaved in 22 nt-long double-stranded molecules by a complex that includes another RNase-III, Dicer. MiRNAs are then ready to be loaded on the RNA-induced silencing complex (RISC): the guide strand is loaded while the star strand is generally degraded [4]. MiRNAs recognize target sequences at the 3' untranslated region (3'-UTR) and repress target gene expression either by targeting the mRNA for degradation or by mediating translation inhibition [5].

A number of miRNAs have been characterized as myogenic modulators (Figure 1). Along well-characterized miRNAs important for muscle homeostasis, during the last few years new studies have pointed out at novel players. MiR-1 and miR-133 are wellestablished members of the so-called myomiRNA family and orchestrate skeletal and cardiac muscle regeneration [6]. Although encoded from the same loci and transcribed together, mir-1 and mir-133 develop into two separate mature miRNAs that hold specific functions. Via the regulation of SRF and MEF2, in fact, they establish distinct negative feedback loops that intrinsically modulate the cellular proliferation and differentiation within muscle cell linages [7]. Mir-206 has also been well characterized as a positive regulator of the myogenic commitment, by supporting satellite cell differentiation and repressing many negative modulators of skeletal muscle differentiation [8]. Its involvement in muscle diseases, for example muscular dystrophies (MDs), has been equally identified [9-11]. In several muscle disorders, including MDs, miRNAs levels are altered. For instance regenerative $m i R-31, m i R-206, m i R-449$ are induced in animal 
models ( $m d x$ mouse) and in Duchenne MD patients during the regenerative stage, while other miRNAs, such as $m i R-1$ and $m i R-29 c$, are down-modulated during fiber degeneration. These observations suggest that the regenerative miRNAs boost differentiation towards myofibers, while during fiber degeneration other miRNAs negatively regulate apoptotic pathway as a compensatory mechanism for myofiber loss [12]. Evidence is also accumulating for a set of non-muscle-specific miRNAs that have an effect on muscle remodeling by targeting muscle-specific regulatory factors. An important difference in contrast to mir-1, mir-133 and mir-206, whose expression is muscle specific, is that these miRNAs are broadly expressed in several tissues and yet retain muscle-specific functions [13]. An example is $m i R-181$, a broadly expressed miRNA that has been shown to contribute to myoblast differentiation by targeting a MyoD repressor in mammals [14]. Mir-181 family comprises six different mature products that are involved in many biological processes, including vascular development, apoptosis, and immunological response among others. For instance, mir-181a increases immature T-cell sensitivity and direct positive and negative selection, intrinsically tuning T-cell antigen recognition [15]. Furthermore, it has been shown that mir-181 family has a role in several cancer types: mir $181 \mathrm{~b}$ is overexpressed in prostate cancer and cervical cancer, while mir-181a is upregulated in ovarian and breast cancer [16-18].

Similarly, mir-24, although not a traditional muscle-specific miRNA, plays a role in positively modulating myogenesis via inhibition of members of the TGF-beta family [19]. Interestingly, mir-24 is expressed at a later stage of muscle differentiation, suggesting an additional important function in the maintenance of the skeletal and cardiac muscle homeostasis.

Along the aforementioned miRNAs, other studies have recently reported the role of additional miRNA regulators. In the cardiac muscle, new evidence has emerged that the regeneration process is additionally (dys)regulated by miRNAs. Mir-128 has a role in cardiac hyperplasia and deposition of extracellular matrix [20]. Moreover, it has been attributed with an anti-myogenic role also in the skeletal muscle, as overexpression of this miRNA inhibits cell proliferation [21]. In addition, the importance of another cluster of miRNAs, the mir-17-92 cluster, has recently emerged. Mir-17-92 cluster is required and sufficient to induce cardiomyocyte proliferation, and mir-19a has been pointed out as the major player within the cluster ${ }^{[22,23]}$. For the skeletal myogenesis, many studies have suggested that mir-29 is a positive regulator of myogenesis. This ubiquitous miRNA is involved in myogenesis by repressing Polycomb silencing complex genes and thereby influencing the chromatin state of early myogenesis genes [24, 25]. Additionally, another study has revealed a more direct target of mir-29, namely $A k t 3$, which is a tyrosine kinase responsive to growth factor signaling, thus linking $m i R-29$ to myoblast differentiation via muscle growth mechanisms [26]. Another remarkable example of fate-driving miRNAs consists of miR-669a/q, which normally repress $M y o D$ in murine cardiomyogenic cells. Dystrophic cardiomyopathic mice displayed depletion of $m i R-669 a / q$ levels and aberrant commitment of cardiac mesoangioblasts [27]. Also, long-term over-expression of the missing miRNA in the myocardium of these mice alleviated end-stage cardiomyopathy and increased survival [28]. 
Thus, unconventional miRNAs are gathering increasingly more attention in achieving complex muscle regulation. However, more studies are required in order to enlighten the still obscure relationships within the muscle-specific endogenous miRNA circuitries. Also, it will be fundamental to elucidate the cellular players potentially involved in mediating the muscular phenotypes induced by these miRNAs.

Recently, several studies have investigated the roles of exosomes in mediating cell-to-cell interactions. Interestingly for this review, exosomal communication has been further associated with skeletal and cardiac muscle remodeling. Exosomes are extracellular vesicles, matured from multivesicular bodies and loaded with a cargo of proteins and different types of RNAs, which are then trafficked in the circulation in steady state and upon induction [29, 30]. MiRNAs can be carried in the circulation either via exosomes, or via non-vesicular carriers, namely protein- or lipoprotein-complexes [31, 32]. Regardless of the carrier nature, the circulating miRNAs are definitely gaining momentum in the study of long-range signals for muscle regulation [32, 33]. If specific miRNAs are enriched in both muscle types and if myocytes shed circulating miRNAs, it is expectable to trace muscle-specific miRNAs in serum or plasma upon striated muscle injuries. Indeed, mir-1, mir-133 and mir-206 are found in body fluids of animal models with skeletal or cardiac degenerative diseases and such observations have further been confirmed in patients [34-36]. Furthermore, a few studies have reported that exosomes secreted during muscle cell differentiation carry fundamental myomiRNAs, suggesting the importance of exosomal miRNAs in orchestrating intracellular communication [37, 38]. Similarly, paracrine action of exosomes secreted by stem cells has been reported, pointing at exosomes as crucial agents in tissue regeneration elicited by cell therapy [39, 40]. For instance, exosomes produced by mesenchymal stem cells promote skeletal muscle repair through activation of angiogenesis and myogenesis pathways [40]. Moreover, exosomes secreted by cardiosphere-derived cells have been identified as pivotal mediators in improvement and regeneration of the infarcted murine heart [39]. Exosome-borne miRNAs are definitely gaining momentum as putative biomarkers, prognostic markers and shuttling signals. However, it is important to further investigate the causal relationships in the exosome-mediated processes. This will be indeed crucial to better discriminate between trafficking exosomes and passively released exosomes in the context of striated muscles.

\section{Signaling pathways regulating muscle growth}

Muscle functions are controlled by signaling pathways that drive muscle response to metabolic and physiological changes, and guide regeneration (Figure 1). The muscle per $s e$ is a metabolic tissue with paracrine properties. Along with hormones and external regulatory factors, myokines -i.e. cytokines produced and released from the skeletal muscle- contribute to maintaining homeostasis and are also involved in the process of myogenesis [41]. Muscle remodeling is a dynamic process occurring throughout the whole tissue life span. During development, protein synthesis and progenitor activation 
contribute to new muscle formation. Conversely, during adulthood, cellular turnover generally decreases and the muscle mass plasticity is mainly determined by the interplay between anabolism and catabolism [42]. Muscular atrophy and muscular hypertrophy are therefore two processes that intertwine in muscle remodeling [43]. The IGF1/PI3K/Akt signaling pathway is involved in muscle hypertrophy in both skeletal and cardiac muscle $[44,45]$. In particular the $A k t / m T O R$ signaling pathway is upregulated during hypertrophy and downregulated during atrophy, indicating its crucial role in maintaining muscle homeostasis [44]. Binding of IGF to its receptor IGFR causes a downstream phosphorylation cascade, which results in activation of phosphatidylinositol-3-kinase PI3K and the subsequent activation of Akt [46]. Equally important for muscle hypertrophy is the HGF-Met signaling pathway. Very interestingly, Met, the high affinity receptor of HGF, is expressed on satellite cells, amongst other several cell types [47, 48]. Transgenic mice expressing a chimeric genetically engineered protein derived from $H G F$ (MAGIC-F1) were generated [49, 50]. Such mice constitute a murine hypertrophic model and display hypertrophy in the heart and in the skeletal muscle.

Myostatin is one of the most powerful negative regulators of muscle growth. In skeletal muscle, myostatin negatively regulates muscle mass through interference with Akt signaling pathways. Recent evidence has shown that, together with myostatin, other members of the TGF $\beta$ family might be involved in regulating skeletal muscle mass and differentiation. In particular, activin-A has been found to be upregulated in skeletal muscle after activation of the tumor necrosis factor alpha/TAK-1 signaling pathway [51]. Furthermore, in the past few years, new signaling players have been unraveled in muscle regeneration. Modulation of G-coupled proteins has been reported to promote skeletal muscle hypertrophy and accelerate skeletal muscle regeneration [52, 53]. Additionally, interest in the inflammatory pathways involved in muscular disorders has been growing, because chronic inflammation is a hallmark of many muscle degenerative conditions, including MDs. Interleukin-6 (IL-6) is, for example, an essential regulator of satellite cell-mediated hypertrophic muscle growth, as it is transiently produced by myofibers and it increases hypertrophic response [54]. Furthermore downstream IL-6, STAT3 signaling has been shown to directly control satellite cell expansion and it has been associated with repair of the skeletal myofibers [55]. Much less is known on the involvement of the aforementioned pathways in cardiac regeneration. $T G F \beta$ family proteins have been involved in modulating the critical regenerative steps following myocardial infarction and have therefore been studied as potential cardiac regeneration modulators [56, 57]. Interestingly for heart regeneration, the recently investigated Hippo/Yap pathway has been found to play essential roles in the regulation of heart development and postnatal cardiomyocyte proliferation through several mechanisms. Yap can interact with different pathways, ranging from $W n t$ pathway, through stimulation via $\beta$-catenin and TCF complex, to interaction with IGF signaling [45, 58-60].

Notch signaling pathway is a major player in regulating skeletal and cardiac myogenesis, and regeneration. Not only is the Notch pathway important during development, but its crucial function in maintaining adult somatic stem cells and driving their fate makes it a key factor in the study of muscle regeneration [61, 62]. Notch signaling is a conserved 
pathway that relies on intracellular ligands and receptors exposed by cells in proximal physical contact. Conventionally, the major ligands are D111/3/4, Jagged1/2 and there are four receptors, Notch1-4. Recently, several pieces of evidence have shown the involvement of Notch signaling in promoting satellite cell self-renewal and myogenic regenerative capacity [63, 64]. Moreover, very recent results have reported the effect of Notch signaling on non-satellite resident myogenic stem cells, showing the pivotal role of Notch in driving cell fate of murine and human mesoangioblasts [65]. Notch signaling plays a predominant role in the cardiac muscle as well, both in the developing heart and as a critical determinant of cardiac stem cell proliferation and differentiation [66-68]. Quite recently, an in vivo study has reported the importance of the activation of Notch signaling pathway after transplantation in order to maintain and ameliorate cell engraftment [69].

Metabolism- and energy-related signals, both circulating and local, are emerging as complex remodelers of the myogenic cell/tissue programs. Ghrelin, for instance, is a gastric hormone stimulating growth hormone release and positive energy balance. Both forms of circulating ghrelin, acylated and unacylated, have been demonstrated to promote myoblast differentiation [70] and to counteract muscle atrophy in mice [71] through p38dependent mechanisms, albeit independently from its conventional receptor [72].

Skeletal muscle is a highly metabolic and energy-requiring tissue, thus mitochondrial function is pivotal in maintaining energy balance and muscle homeostasis. Mitochondrial dysfunction can therefore also affect muscle and its regeneration. Mutations that cause impairment of mitochondrial fusion results in severe mitochondrial DNA depletion and muscle abnormalities, such as atrophy [75]. Mitochondrial myopathies are marked by perturbations in mitochondrial biogenesis, content and function, leading to muscle weakness and functionality loss. To this regard, studies have reported that exercise training might improve muscle function of patients with mitochondrial DNA mutations by activation of specific stress signals that act as positive modulators of crucial transcriptional pathways implicated in skeletal muscle mitochondrial biogenesis, fusion and metabolism [76, 77]. Furthermore, increasing evidence points at dysfunctional mitochondria as hubs of impaired signaling based on disrupted energy transfer/sensing. An example has been firstly reported in the case of $M L P$-null transgenic mice, displaying regional absence of mitochondria and thereby disturbed energy balance in the myocardium, leading to dilated cardiomyopathy [73]. Interestingly, skeletal muscle mitochondrial dysfunction has been associated with metabolic diseases, such as type 2 diabetes, under inactive conditions [74].

Therefore, manipulating the signaling pathways still constitutes a fascinating perspective for regulating the myogenic paths, often in clinically relevant conditions. However, many questions are still open on how the diverse pathways intertwine with each other in cardiac and skeletal muscles, and in the different stem cell pools. In this view, it will be important to dissect the cellular players and the signal directionalities involved in diseased/regenerating striated muscles. 


\section{Epigenetic regulators: adding up on myogenic complexity}

Not only do genetic factors influence cell fate decision and myogenesis in the adult skeletal and cardiac muscle, but it is increasingly evident that there is a non-negligible level of cooperative regulation carried out by epigenetic mechanisms, transcription factors and signaling [78]. Epigenetic factors, such as chromatin remodeling, histone mark modifications and DNA methylation, play therefore an important role in regulating muscle gene expression. Muscle transcription factors, e.g. MyoD and $M E F 2$, are regulated by histone deacetylases in undifferentiated myoblasts to prevent unscheduled activation, while upon differentiation such complexes are displaced and histones are accessible for activating modifications, i.e. acetylation of H3K27 and trimethylation of H3K4 [79-81]. Closely linked to histone mark modification is the DNA methylation machinery regulating $M y o D$. This layer of expression control was firstly observed when treatment with a DNA methyltransferase inhibitor, 5-azacytidine, converted 10T1/2 embryonic fibroblasts to myoblasts [82].

Although not many studies have been performed on quiescent satellite cells, it is generally accepted that skeletal muscle satellite cells are kept in quiescent state also by means of epigenetic cues, DNA methylation and repressive histone marks [83]. When the muscle is injured, satellite cells are activated. In this state, cell cycle genes and early myogenic fate markers are characterized by permissive histone marks, whereas quiescence markers, such as $\operatorname{Pax} 7$, are silenced by repressive marks [83]. Recent evidence has reported the presence of poised domains in quiescent satellite cells, where chromatin carries bivalent histone marks. Several genes, including important myogenic regulatory factors, are marked by permissive histone marks, such as H3K4me3, indicating that satellite cells are primed for future myogenic differentiation already at a quiescent state by keeping key genes under poised chromatin state [84]. Polycomb groups and thritorax groups' proteins have been largely implicated in the modulation of histone status during myogenesis and muscle regeneration $[85,86]$. These two histone methyltransferase complexes act in opposite ways, as they respectively establish low levels and high levels of transcription on the same regulatory genes. In the skeletal muscle, Ezh1 and Ezh2, proteins of the polycomb complex, are responsible for epigenetic regulation of satellite cells and support of their expansion and early differentiation, but are also interestingly required for the homeostasis of the adult satellite cell pool $[85,87$, 88]. Additionally, as a possible link between chromatin modification and DNA methylation, Ezh2 was shown to target the de novo DNA methyltransferase enzymes, $D N M T 3 a$ and $D N M T 3 b$, to specific genes [89]. Interestingly, a recent study has shown the involvement of Ezh2 and DNMT3b in pathological conditions: in Duchenne MD both factors cooperate to achieve repression of the transcription at the Notch 1 promoter during satellite cell activation, thus causing repression of the muscular regenerative capacity [90]. Intriguingly, novel evidence has reported that several genes involved in Notch signaling pathway hold hypomethylated and hydroxymethylated intragenic or intergenic regions in myoblasts and myotubes [91]. 
Not much is known with regards to the epigenetic factors regulating cardiac regeneration. It is however established that several cardiovascular diseases are associated with epigenetic modifications. Heart failure is reportedly marked by abnormal DNA methylation and altered gene expression of angiogenic factors. Conversely, the histone acetyltransferases domain of $\mathrm{p} 300$, which acetylates transcription factors such as GATA4, is involved in promoting LV remodeling after myocardial infarction. This aspect emerges from the observation of cardiovascular system abnormality and depressed cardiac dilation in transgenic mice overexpressing intact $\mathrm{p} 300$ in the heart, but not in mice overexpressing a mutant p300, lacking its histone acetyltransferase activity [92, 93]. ATP-dependent chromatin remodeling complexes have been implicated in cardiac development and function, especially those of the SWI/SNF family. These complexes can remove, add or displace nucleosomes using ATP [94]. During the development of the cardiac muscle, several epigenetic mechanisms have an important role in timing the expression of regulatory cardiac genes. Histone acetyltransferases allow timing of the expression of regulatory genes, histone deacetylases have a role in remodeling, while characteristic DNA methylation patterns establish regulatory circuits. However, whether these developmental regulatory elements can be relevant for cardiac regeneration therapies still remains to be elucidated in future studies.

\section{Perspectives}

In conclusion, the study of unconventional non-genetic factors in myogenesis is contributing to deciphering striated muscle complexity, while amassing novel intriguing questions. An important question substantially still open is which and how non-genetic signals overlap in determining cardiac vs skeletal myogenic routes, particularly in humans. Systematic approaches in this perspective, likely with the help of advanced bidirectional cell systems, will definitely improve our knowledge and handling of the two myogenic routes, consolidating previous translational approaches. A second intriguing perspective consists in the tantalizing question of where/when the different non-genetic cues converge in specifying the myogenic fates. In this context, a shift from anecdotic to comprehensive paradigms is needed in order to increase the complexity of interpretation for future findings on striated muscle regeneration.

\section{Acknowledgements}

We would like to apologize to all authors whose work has not been reported here due to space limitations. This work has been supported with the contribution of "Opening The Future" Campaign (EJJ-OPTFUT-02010), CARE-MI FP7, AFM Telethon, CARIPLO, FWO, GOA, IUAP and OT grants. MQ is supported by an FWO Postdoctoral Fellowship. We thank Christina Vochten and Vicky Raets for professional administrative 
assistance. We would also like to thank Paolo Luban and Rondoufonds voor Duchenne Onderzoek for kind donations. 


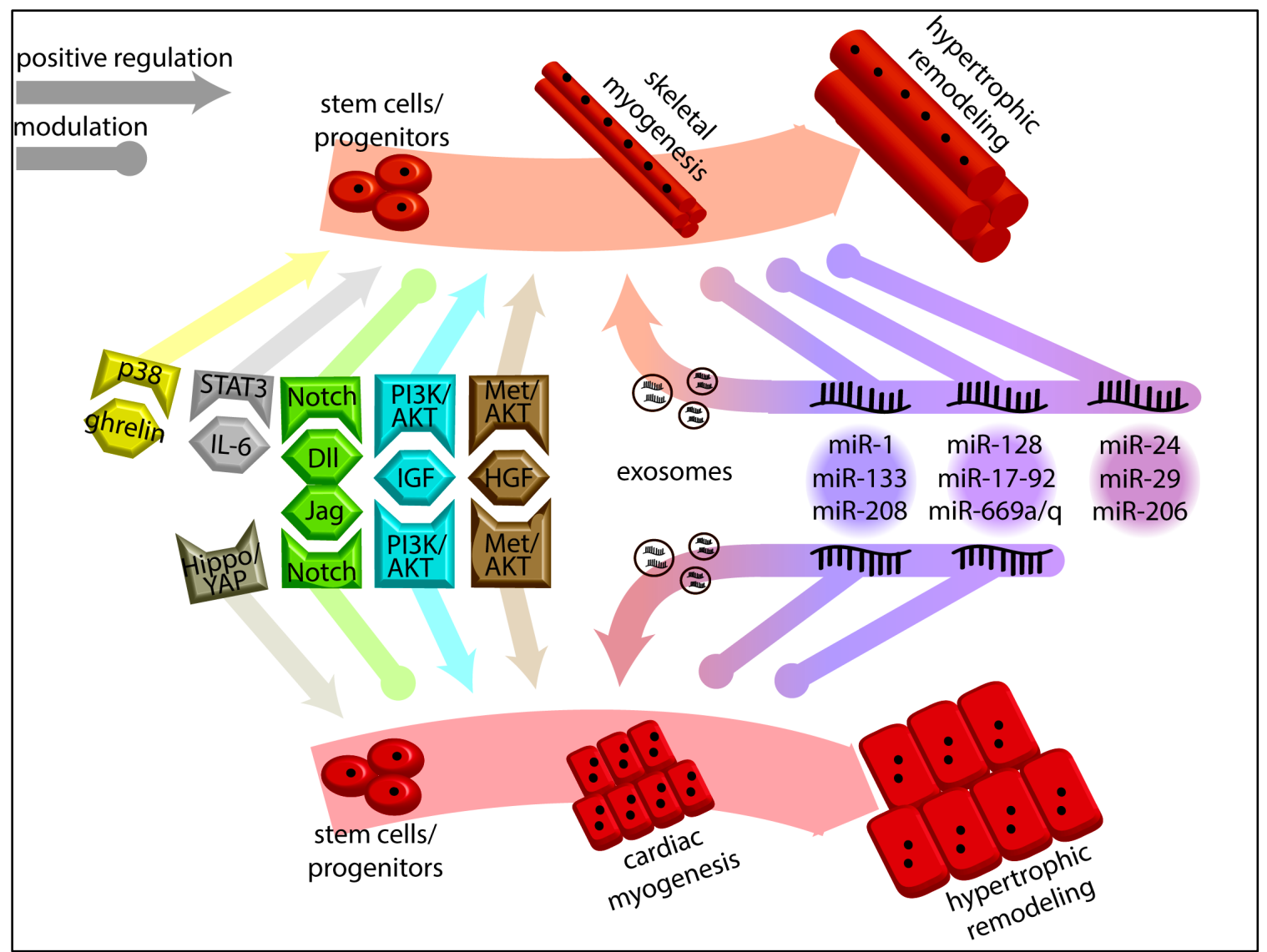

Figure 1 - Schematic representation of several non-genetic myogenic modulators reported in the review. Ligands and downstream signaling pathways are reported on the left, whereas miRNAs, possibly through exosome-mediated trafficking, are listed on the right. Arrow-capped connectors represent positive regulation, whereas circle-capped connectors indicate complex modulation. 


\section{References}

1. Shenoy, A. and R.H. Blelloch, Regulation of microRNA function in somatic stem cell proliferation and differentiation. Nature reviews Molecular cell biology, 2014.

2. Westholm, J.O. and E.C. Lai, Mirtrons: microRNA biogenesis via splicing. Biochimie, 2011. 93(11): p. 1897-1904.

3. Ha, M. and V.N. Kim, Regulation of microRNA biogenesis. Nature reviews Molecular cell biology, 2014. 15(8): p. 509-524.

4. Gregory, R.I., et al., Human RISC couples microRNA biogenesis and posttranscriptional gene silencing. Cell, 2005. 123(4): p. 631-640.

5. Djuranovic, S., A. Nahvi, and R. Green, miRNA-mediated gene silencing by translational repression followed by mRNA deadenylation and decay. Science, 2012. 336(6078): p. 237-240.

6. Chen, J.-F., et al., The role of microRNA-1 and microRNA-133 in skeletal muscle proliferation and differentiation. Nature genetics, 2005. 38(2): p. 228-233.

7. Liu, N., et al., An intragenic MEF2-dependent enhancer directs muscle-specific expression of microRNAs 1 and 133. Proceedings of the National Academy of Sciences, 2007. 104(52): $p$. 20844-20849.

8. Kim, H.K., et al., Muscle-specific microRNA miR-206 promotes muscle differentiation. The Journal of cell biology, 2006. 174(5): p. 677-687.

9. Chen, J.-F., et al., microRNA-1 and microRNA-206 regulate skeletal muscle satellite cell proliferation and differentiation by repressing Pax7. The Journal of cell biology, 2010. 190(5): $p$. 867-879.

10. Cacchiarelli, D., et al., MicroRNAs involved in molecular circuitries relevant for the Duchenne muscular dystrophy pathogenesis are controlled by the dystrophin/nNOS pathway. Cell metabolism, 2010. 12(4): p. 341-351.

11. Townley-Tilson, W., T.E. Callis, and D. Wang, MicroRNAs 1, 133, and 206: critical factors of skeletal and cardiac muscle development, function, and disease. The international journal of biochemistry \& cell biology, 2010. 42(8): p. 1252-1255.

12. Greco, S., et al., Common micro-RNA signature in skeletal muscle damage and regeneration induced by Duchenne muscular dystrophy and acute ischemia. The FASEB Journal, 2009. 23(10): p. 3335-3346.

13. Eisenberg, I., M.S. Alexander, and L.M. Kunkel, miRNAS in normal and diseased skeletal muscle. Journal of cellular and molecular medicine, 2009. 13(1): p. 2-11.

14. Naguibneva, I., et al., The microRNA miR-181 targets the homeobox protein Hox-A11 during mammalian myoblast differentiation. Nature cell biology, 2006. 8(3): p. 278-284.

15. Li, Q.-J., et al., miR-181a is an intrinsic modulator of T cell sensitivity and selection. Cell, 2007. 129(1): p. 147-161.

16. Bryant, R., et al., Changes in circulating microRNA levels associated with prostate cancer. British journal of cancer, 2012. 106(4): p. 768-774.

17. Taylor, M.A., et al., TGF-B upregulates miR-181a expression to promote breast cancer metastasis. The Journal of clinical investigation, 2013. 123(1): p. 150-163.

18. Parikh, A., et al., microRNA-181a has a critical role in ovarian cancer progression through the regulation of the epithelial-mesenchymal transition. Nature communications, 2014. 5.

19. Sun, Q., et al., Transforming growth factor-6-regulated miR-24 promotes skeletal muscle differentiation. Nucleic acids research, 2008. 36(8): p. 2690-2699. 
20. Witman, N., et al., miR-128 regulates non-myocyte hyperplasia, deposition of extracellular matrix and $<$ i> Islet $1</ i>$ expression during newt cardiac regeneration. Developmental biology, 2013. 383(2): p. 253-263.

21. Motohashi, N., et al., Regulation of IRS1/Akt insulin signaling by microRNA-128a during myogenesis. Journal of cell science, 2013. 126(12): p. 2678-2691.

22. Wu, G., Z.-P. Huang, and D.-Z. Wang, microRNAs in cardiac regeneration and cardiovascular disease. Science China Life Sciences, 2013. 56(10): p. 907-913.

23. Chen, J., et al., mir-17-92 cluster is required for and sufficient to induce cardiomyocyte proliferation in postnatal and adult hearts. Circulation research, 2013. 112(12): p. 1557-1566.

24. Sharma, M., et al., Mega roles of microRNAs in regulation of skeletal muscle health and disease. Frontiers in physiology, 2014. 5.

25. Zhou, L., et al., A novel target of microRNA-29, Ring1 and YY1-binding protein (Rybp), negatively regulates skeletal myogenesis. Journal of Biological Chemistry, 2012. 287(30): p. 25255-25265.

26. Wei, W., et al., miR-29 targets Akt3 to reduce proliferation and facilitate differentiation of myoblasts in skeletal muscle development. Cell death \& disease, 2013. 4(6): p. e668.

27. Crippa, S., et al., miR669a and miR669q prevent skeletal muscle differentiation in postnatal cardiac progenitors. The Journal of cell biology, 2011. 193(7): p. 1197-1212.

28. Quattrocelli, M., et al., Long-Term miR-669a Therapy Alleviates Chronic Dilated Cardiomyopathy in Dystrophic Mice. Journal of the American Heart Association, 2013. 2(4): p. e000284.

29. Théry, C., L. Zitvogel, and S. Amigorena, Exosomes: composition, biogenesis and function. Nature Reviews Immunology, 2002. 2(8): p. 569-579.

30. Valadi, H., et al., Exosome-mediated transfer of mRNAs and microRNAs is a novel mechanism of genetic exchange between cells. Nature cell biology, 2007. 9(6): p. 654-659.

31. Atay, S. and A.K. Godwin, Tumor-derived exosomes: A message delivery system for tumor progression. Communicative \& integrative biology, 2014. 7(1).

32. Creemers, E.E., A.J. Tijsen, and Y.M. Pinto, Circulating microRNAs novel biomarkers and extracellular communicators in cardiovascular disease? Circulation research, 2012. 110(3): p. 483-495.

33. Aoi, W. and K. Sakuma, Does regulation of skeletal muscle function involve circulating microRNAs? Frontiers in physiology, 2014. 5.

34. Mizuno, H., et al., Identification of muscle-specific microRNAs in serum of muscular dystrophy animal models: promising novel blood-based markers for muscular dystrophy. PloS one, 2011. 6(3): p. e18388.

35. Roberts, T.C., et al., Expression analysis in multiple muscle groups and serum reveals complexity in the microRNA transcriptome of the $m d x$ mouse with implications for therapy. Molecular Therapy-Nucleic Acids, 2012. 1(8): p. e39.

36. Cacchiarelli, D., et al., miRNAs as serum biomarkers for Duchenne muscular dystrophy. EMBO molecular medicine, 2011. 3(5): p. 258-265.

37. Forterre, A., et al., Myotube-derived exosomal miRNAs downregulate Sirtuin 1 in myoblasts during muscle cell differentiation. Cell Cycle, 2013. 13(1): p. 78-89.

38. Forterre, A., et al., Proteomic Analysis of C2C12 Myoblast and Myotube Exosome-Like Vesicles: A New Paradigm for Myoblast-Myotube Cross Talk? PloS one, 2014. 9(1): p. e84153.

39. Ibrahim, A.G.-E., K. Cheng, and E. Marbán, Exosomes as Critical Agents of Cardiac Regeneration Triggered by Cell Therapy. Stem Cell Reports, 2014. 2(5): p. 606-619.

40. Lai, R.C., T.S. Chen, and S.K. Lim, Mesenchymal stem cell exosome: a novel stem cell-based therapy for cardiovascular disease. Regenerative medicine, 2011. 6(4): p. 481-492.

41. Pedersen, B.K., Muscles and their myokines. The Journal of experimental biology, 2011. 214(2): p. 337-346. 
42. Cassano, M., et al., Cellular mechanisms and local progenitor activation to regulate skeletal muscle mass. Journal of muscle research and cell motility, 2009. 30(7-8): p. 243-253.

43. Glass, D.J., Signalling pathways that mediate skeletal muscle hypertrophy and atrophy. Nature cell biology, 2003. 5(2): p. 87-90.

44. Bodine, S.C., et al., Akt/mTOR pathway is a crucial regulator of skeletal muscle hypertrophy and can prevent muscle atrophy in vivo. Nature cell biology, 2001. 3(11): p. 1014-1019.

45. Lin, Z., et al., Pi3kcb Links Hippo-YAP and PI3K-AKT Signaling Pathways to Promote Cardiomyocyte Proliferation and Survival. Circulation research, 2014: p. CIRCRESAHA. 114.304457.

46. Schiaffino, S. and C. Mammucari, Regulation of skeletal muscle growth by the IGF1-Akt/PKB pathway: insights from genetic models. Skelet Muscle, 2011. 1(4): p. 232-6.

47. Sheehan, S.M., et al., HGF is an autocrine growth factor for skeletal muscle satellite cells in vitro. Muscle \& nerve, 2000. 23(2): p. 239-245.

48. Miller, K.J., et al., Hepatocyte growth factor affects satellite cell activation and differentiation in regenerating skeletal muscle. American Journal of Physiology-Cell Physiology, 2000. 278(1): p. C174-C181.

49. Cassano, M., et al., Magic-factor 1, a partial agonist of Met, induces muscle hypertrophy by protecting myogenic progenitors from apoptosis. PloS one, 2008. 3(9): p. e3223.

50. Ronzoni, F., et al., Localization of Magic-F1 Transgene, Involved in Muscular Hypertrophy, during Early Myogenesis. BioMed Research International, 2011. 2011.

51. Trendelenburg, A.U., et al., TAK-1/p38/nNF B signaling inhibits myoblast differentiation by increasing levels of Activin A. Skeletal muscle, 2012. 2(1).

52. Minetti, G.C., et al., G \{alpha\} i2 Signaling Promotes Skeletal Muscle Hypertrophy, Myoblast Differentiation, and Muscle Regeneration. Science signaling, 2011. 4(201): p. ra80.

53. Minetti, G.C., et al., Gai2 Signaling Is Required for Skeletal Muscle Growth, Regeneration, and Satellite Cell Proliferation and Differentiation. Molecular and cellular biology, 2014. 34(4): p. 619-630.

54. Serrano, A.L., et al., Interleukin-6 is an essential regulator of satellite cell-mediated skeletal muscle hypertrophy. Cell metabolism, 2008. 7(1): p. 33-44.

55. Tierney, M.T., et al., STAT3 signaling controls satellite cell expansion and skeletal muscle repair. Nature medicine, 2014. 20(10): p. 1182-1186.

56. Bujak, M. and N.G. Frangogiannis, The role of TGF-6 signaling in myocardial infarction and cardiac remodeling. Cardiovascular research, 2007. 74(2): p. 184-195.

57. Goumans, M.-J., et al., TGF-B1 induces efficient differentiation of human cardiomyocyte progenitor cells into functional cardiomyocytes < i> in vitro</i>. Stem Cell Research, 2008. 1(2): p. 138-149.

58. Heallen, T., et al., Hippo pathway inhibits Wnt signaling to restrain cardiomyocyte proliferation and heart size. Science, 2011. 332(6028): p. 458-461.

59. Xin, M., et al., Hippo pathway effector Yap promotes cardiac regeneration. Proceedings of the National Academy of Sciences, 2013. 110(34): p. 13839-13844.

60. Xin, M., et al., Regulation of insulin-like growth factor signaling by Yap governs cardiomyocyte proliferation and embryonic heart size. Science signaling, 2011. 4(196): p. ra70.

61. Conboy, I.M. and T.A. Rando, The regulation of Notch signaling controls satellite cell activation and cell fate determination in postnatal myogenesis. Developmental cell, 2002. 3(3): p. 397-409.

62. Luo, D., V.M. Renault, and T.A. Rando. The regulation of Notch signaling in muscle stem cell activation and postnatal myogenesis. in Seminars in cell \& developmental biology. 2005.

Elsevier. 
63. Wen, Y., et al., Constitutive Notch activation upregulates Pax7 and promotes the self-renewal of skeletal muscle satellite cells. Molecular and cellular biology, 2012. 32(12): p. 2300-2311.

64. Mourikis, P., et al., Cell-autonomous Notch activity maintains the temporal specification potential of skeletal muscle stem cells. Development, 2012. 139(24): p. 4536-4548.

65. Quattrocelli, M., et al., Notch signaling regulates myogenic regenerative capacity of murine and human mesoangioblasts. Cell death \& disease, 2014. 5(10): p. e1448.

66. Collesi, C., et al., Notch1 signaling stimulates proliferation of immature cardiomyocytes. The Journal of cell biology, 2008. 183(1): p. 117-128.

67. Zhao, L., et al., Notch signaling regulates cardiomyocyte proliferation during zebrafish heart regeneration. Proceedings of the National Academy of Sciences, 2014. 111(4): p. 1403-1408.

68. Boni, A., et al., Notch1 regulates the fate of cardiac progenitor cells. Proceedings of the National Academy of Sciences, 2008. 105(40): p. 15529-15534.

69. Parker, M.H., et al., Activation of Notch signaling during ex vivo expansion maintains donor muscle cell engraftment. Stem Cells, 2012. 30(10): p. 2212-2220.

70. Filigheddu, N., et al., Ghrelin and des-acyl ghrelin promote differentiation and fusion of C2C12 skeletal muscle cells. Molecular biology of the cell, 2007. 18(3): p. 986-994.

71. Porporato, P.E., et al., Acylated and unacylated ghrelin impair skeletal muscle atrophy in mice. The Journal of clinical investigation, 2013. 123(2): p. 611-622.

72. Reano, S., A. Graziani, and N. Filigheddu, Acylated and unacylated ghrelin administration to blunt muscle wasting. Current Opinion in Clinical Nutrition \& Metabolic Care, 2014. 17(3): p. 236-240.

73. van den Bosch, B.J., et al., Regional absence of mitochondria causing energy depletion in the myocardium of muscle LIM protein knockout mice. Cardiovascular research, 2005. 65(2): p. 411418.

74. Van Tienen, F., et al., Physical activity is the key determinant of skeletal muscle mitochondrial function in type 2 diabetes. The Journal of Clinical Endocrinology \& Metabolism, 2012. 97(9): p. 3261-3269.

75. Chen, H., et al., Mitochondrial fusion is required for mtDNA stability in skeletal muscle and tolerance of mtDNA mutations. Cell, 2010. 141(2): p. 280-289.

76. Taivassalo, T., et al., Endurance training and detraining in mitochondrial myopathies due to single large-scale mtDNA deletions. Brain : a journal of neurology, 2006. 129(12): p. 3391-3401.

77. Adhihetty, P.J., et al., The effect of training on the expression of mitochondrial biogenesis-and apoptosis-related proteins in skeletal muscle of patients with mtDNA defects. American Journal of Physiology-Endocrinology and Metabolism, 2007. 293(3): p. E672-E680.

78. Giordani, L. and P.L. Puri, Epigenetic control of skeletal muscle regeneration. FEBS Journal, 2013. 280(17): p. 4014-4025.

79. Puri, P.L., et al., Class I histone deacetylases sequentially interact with MyoD and pRb during skeletal myogenesis. Molecular cell, 2001. 8(4): p. 885-897.

80. Zhang, C.L., T.A. McKinsey, and E.N. Olson, Association of class II histone deacetylases with heterochromatin protein 1: potential role for histone methylation in control of muscle differentiation. Molecular and cellular biology, 2002. 22(20): p. 7302-7312.

81. Mal, A., et al., A role for histone deacetylase HDAC1 in modulating the transcriptional activity of MyoD: inhibition of the myogenic program. The EMBO journal, 2001. 20(7): p. 1739-1753.

82. Taylor, S.M. and P.A. Jones, Changes in phenotypic expression in embryonic and adult cells treated with 5-azacytidine. Journal of cellular physiology, 1982. 111(2): p. 187-194.

83. Perdiguero, E., et al., Epigenetic regulation of myogenesis. Epigenetics, 2009. 4(8): p. 541-550.

84. Liu, L., et al., Chromatin modifications as determinants of muscle stem cell quiescence and chronological aging. Cell reports, 2013. 4(1): p. 189-204. 
85. Caretti, G., et al., The Polycomb Ezh2 methyltransferase regulates muscle gene expression and skeletal muscle differentiation. Genes \& development, 2004. 18(21): p. 2627-2638.

86. Schuettengruber, B. and G. Cavalli, Recruitment of polycomb group complexes and their role in the dynamic regulation of cell fate choice. Development, 2009. 136(21): p. 3531-3542.

87. Woodhouse, S., et al., Ezh2 maintains a key phase of muscle satellite cell expansion but does not regulate terminal differentiation. Journal of cell science, 2013. 126(2): p. 565-579.

88. Juan, A.H., et al., Polycomb EZH2 controls self-renewal and safeguards the transcriptional identity of skeletal muscle stem cells. Genes \& development, 2011. 25(8): p. 789-794.

89. Viré, E., et al., The Polycomb group protein EZH2 directly controls DNA methylation. Nature, 2005. 439(7078): p. 871-874.

90. Acharyya, S., et al., TNF inhibits Notch-1 in skeletal muscle cells by Ezh2 and DNA methylation mediated repression: implications in duchenne muscular dystrophy. PloS one, 2010. 5(8): $p$. e12479.

91. Terragni, J., et al., Notch signaling genes: Myogenic DNA hypomethylation and 5hydroxymethylcytosine. Epigenetics, 2014. 9(6): p. 0--1.

92. Miyamoto, S., et al., Histone acetyltransferase activity of $p 300$ is required for the promotion of left ventricular remodeling after myocardial infarction in adult mice in vivo. Circulation, 2006. 113(5): p. 679-690.

93. Baccarelli, A., et al., Ischemic heart disease and stroke in relation to blood DNA methylation. Epidemiology (Cambridge, Mass.), 2010. 21(6): p. 819.

94. Han, P., et al., Chromatin remodeling in cardiovascular development and physiology. Circulation research, 2011. 108(3): p. 378-396. 